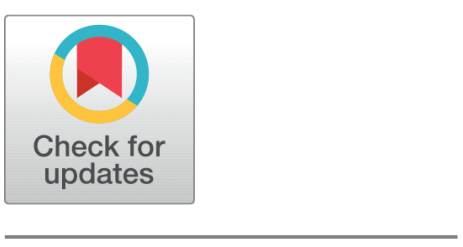

OPEN ACCESS

Received: 03.08.2021

Accepted: 18.09 .2021

Published: 11.10 .2021

Citation: Matta JCP, Siddiah P (2021) A Modified OMP Algorithm with Reduced Feedback Overhead for Massive MIMO System. Indian Journal of Science and Technology 14(33): 2663-2670. https://doi.org/ 10.17485/IJST/v14i33.1442

* Corresponding author. jagadishmatta@gmail.com

Funding: None

Competing Interests: None

Copyright: (c) 2021 Matta \& Siddiah. This is an open access article distributed under the terms of the Creative Commons Attribution License, which permits unrestricted use, distribution, and reproduction in any medium, provided the original author and source are credited.

Published By Indian Society for Education and Environment (iSee)

ISSN

Print: 0974-6846

Electronic: 0974-5645

\section{A Modified OMP Algorithm with Reduced Feedback Overhead for Massive MIMO System}

\author{
Jagadeesh Chandra Prasad Matta ${ }^{1 *}$, P Siddiah $^{2}$ \\ 1 Research Scholar, ECE Department University College of Engineering \& Technology, Acarya \\ Nagarjuna University, Guntur, Andhra Pradesh, India \\ 2 Professor in ECE Department, University College of Engineering \& Technology, Acarya \\ Nagarjuna University, Guntur, Andhra Pradesh, India
}

\section{Abstract}

Objectives: Massive Multiple-Input and Multiple-Output (MIMO) is the optimum way to enhance the bandwidth issue, in which the feedback overhead is a challenging concern when tested with Frequency Division Duplex (FDD) systems. Conventional greedy search algorithms like Matching Pursuit (MP) and Orthogonal Matching Pursuit (OMP) are based on the prior knowledge of the signal sparsity, which enhances overhead. So, the objective of this study is to develop a method with low complexity channel estimation and reduced feedback overhead. Methods: The proposed method is based on the residual vector which is updated after iteration and a threshold-based approach is utilized to decide the stopping criteria thereby controlling the number of iterations. The evaluation parameters used in the experimental analysis are Normalized Mean Square Error and channel capacity. Findings: The proposed method improves the effectiveness in signal recovery and reconstruction along with signal sparsity it also reduces the complexity of the estimation process and limits the feedback. This results in a high data rate and can be used in a massive MIMO system to optimize the channel estimation. Novelty: The proposed model is independent of the sparsity in the input vector and improves the effectiveness of the signal recovery and reduces the feedback burden. The proposed method is suitable for a $5 \mathrm{G}$ massive MIMO systems.

Keywords: Feedback overhead; CSI; OMP; Residue vector

\section{Introduction}

The Massive Multiple-Input Multiple-Output (MIMO) system is equipped with a large array of antennas in the base station, which provides a high degree of freedom of space. This can effectively improve energy efficiency and spectrum efficiency, and become one of the key technologies in the $5 \mathrm{G}$ mobile communication ${ }^{(1,2)}$. To avail the advantage of the spatial gain of a massive MIMO system, the channel state information of the sender is necessary. In the time division duplexing (TDD) system, the downlink channel status information can be transferred from the uplink channel state information (CSI) 
according to the reciprocity of the channel. However, in the TDD system, the frequency calibration error of the radio frequency link is relatively large. Generally, it is impossible to obtain accurate $\mathrm{CSI}^{(3)}$. But in the FDD system, Channel reciprocity does not exist, FDD system downlink can be directly transferred from the uplink channel. For channel estimation, the uplink requires channel feedback technology. Therefore, we study the massive MIMO system in FDD mode.

The uplink channel estimation and uplink channel feedback schemes have high research investigation value. Channel training and channel feedback schemes are mainly divided into three categories:

1. Limited feedback technology based on channel statistics

2. Codebook-based Limited feedback technology

3. Limited feedback technology based on compressed sensing

In limited feedback technology ${ }^{(4)}$ and the codebook based feedback technology, accuracy is low and the complexity is high. Based on the compressed sensing method Channel estimation and feedback scheme can effectively decrease system feedback overhead.

In recent years, limited feedback technology methodologies based on compressed sensing have been proposed ${ }^{(5,6)}$. L. Daietal. ${ }^{(7)}$ first designed a non-orthogonal pilot, and then proposed an adaptive structured compressed sensing algorithm, which effectively improved the channel estimation accuracy, but the feedback link overhead was still large. In ${ }^{(8)}$ compressed sensing channel estimation scheme based on beam block is presented, which effectively reduces the downlink feedback overhead, but it cannot obtain a high channel estimation effect in a limited pilot sequence. W. Shenetal. ${ }^{(9)}$ designed a joint channel estimation and feedback differential channel estimation feedback scheme. The scheme used the characteristics of the channel impulse response of adjacent time slots so that the channel impulse response does not change much, and the differential channel impulse response is used to a certain extent. The channel estimation accuracy is improved, and the feedback scheme effectively reduces the piloting overhead.

Stefan Schwarz et al. ${ }^{(10)}$ proposed a novel CSI quantization and feedback technique based on a dual-stage Grassmannian product quantization framework. The proposed method exhibits better performance particularly when the channel is decomposed in the angular domain. So that, this DFT-based codebook provides efficient CSI compression in the massive MIMO system. The proposed dual-stage codebook design is compared with single-stage quantization and it reveals small degradation in the performance. However, to improve the reduction in feedback overhead in massive MIMO, the development of the hybrid method is considered as a future scope.

According to Feng Zheng et al. ${ }^{(11)}$, dual-polarized antennas are widely used in massive MIMO systems. When these antennas are utilized in massive MIMO, polarization leakage is a critical issue. Most of the existing CSI methods are not considering these polarization issues. In this article, polarization leakage is considered into account and a CSI method is developed. Similarly, two novel CSI feedback schemes were introduced which are: explicit and implicit feedback schemes. In an explicit feedback scheme, the feedback operation performs explicitly to determine the eigenvectors of the channels. In the implicit feedback scheme, the two feedback of the two channels is based on a predetermined codebook.

Haoran Sun et al. ${ }^{(12)}$ proposed two novel approaches for channel estimation to handle the feedback burden in the massive MIMO system. The first approach was developed based on iterative optimization which is a low-rank sensing method. The second approach is based on a deep neural network framework for channel recovery in real-time. These two methods are compared with existing optimization methods to evaluate their effectiveness. The main drawback of the second approach is: the huge computational time is required for training.

Yong Liao et al. ${ }^{(13)}$ uses the spatial correlation of massive MIMO and proposed a channel simulation compression feedback method to obtain better feedback performance with smaller power and shorter time. In this work a CSI compressed feedback algorithm is developed based on Laplacian eigenmaps. The authors reported that, by using the proposed method, the feedback overhead burden is greatly reduced when compared with the Discrete Cosine Transform (DCT) based sparse compression method. However, the computational complexity of this method is increased.

Yong Liao et al. ${ }^{(14)}$ describes the importance of feedback overhead reduction in FDD massive MIMO. To overcome feedback overhead, a Modified Sparse Adaptive Matching Pursuit (M-SAMP) method is proposed. The authors combine the parameters of the conventional SAMP algorithm to precisely construct the sparse signal. The combined considered parameters are variable step size, signal segmenting, and primary estimation of the sparsity signal. This proposed work is compared with other algorithms such as OMP, subspace tracking (SP), and conventional SAMP. However, in this work, quantization error and complexity of the proposed method are not discussed. Darshankumarand Himanshu, ${ }^{(15)}$ presented an approach to improve the channel estimation using the Kalman filter, however, this approach may not be the optimum to find the directivity in realtime applications. Nivedita and Manoj ${ }^{(16)}$ proposed a method to enhance the channel estimation using DWT Technique; this approach cannot find extensive use in MIMO systems because it lacks to find directivity. Ahmed et. al ${ }^{(17)}$ proposed a method 
in which the authors try to improve the downlink performance of massive MIMO systems using the pre-coding technique. However, this method may not be optimum in the real-time application as pre-coding causes overhead and enhances delay, as a result of which PDR is reduced. Lee and Song ${ }^{(18)}$ demonstrated a technique to improve beamforming by reducing the feedback overhead using the nonlinear quantization principle. This technique increases the overhead due to more no of bits combined along with the frame, hence may not be the optimum for real-time applications. Borges et. al ${ }^{(19)}$ gives a survey of Massive MIMO techniques.Mashhadi and Deniz ${ }^{(2)}$ presented a technique in which authors use deep learning concept to optimize the CSI, however, environmental condition varies for seasons, hence cannot be used to improve beamforming which is an essential parameter to improve channel estimation

Since most of the current channel estimation and feedback schemes have the problem of low channel estimation accuracy and large feedback overhead, it is very significant to study algorithms to improve channel estimation accuracy and reduce feedback link overhead. So this paper proposes a low complexity channel estimation algorithm based on the traditional OMP approach which effectively improves the channel estimation and gives a better performance with reference to the packet delivery ratio (PDR). The proposed approach reduces the complexity and reduces the delay, thus is well suited for massive MIMO systems for real-time applications.

The organization of the paper is as follows: In section-2, the conventional greedy algorithms are being presented, in section 3 we present our proposed model, section 4 presents the results and discussion. And we conclude our paper in section 5.

\section{Conventional Greedy Algorithms}

The function of a greedy search algorithm is to sequentially find the support of the signal to be reconstructed through an iterative method, and find one or more constituent elements of the signal to be estimated at a time based on a certain greedy criterion. The greedy algorithm represented by Matching Pursuit (MP) and Orthogonal Matching Pursuit (OMP) searches for the idea of ' $x^{\prime}$ signal support that is, each iteration uses the column with the largest absolute value element $\Phi^{\mathrm{T}} \mathrm{y}$ as the supported candidate, and one iteration determines one selected column vector. The $\mathrm{MP}^{(21)}$ and OMP algorithms are described in algorithms 1 and 2 respectively.

Algorithm 2.1: MP algorithm

- Initialize redundant vector $r_{0}=y$, iteration count $t=1$;

- Find the index entry $\lambda_{t}$,

$$
\lambda_{t}=\arg \min _{j=1,2, \ldots, N}
$$

- Calculate the new approximation $\mathrm{a}_{t}$ and redundant $\mathrm{r}_{t}$ :

$$
a_{t}=\left\langle r_{t-1}, \Phi_{\lambda_{t}}\right\rangle, \quad r_{t}=r_{t}-a_{t}
$$

- $t=t+1$, if $t<k$ return to step 2

The MP algorithm and the OMP algorithm have the same optimal column vector selection strategy. Each iteration column vector with the largest inner product in the measurement matrix $\Phi$ and the current redundant vector $r_{t-1}$ are used as the supported candidate for the signal to be estimated. However, these two different vectors are in the residual update method. The MP algorithm guarantees the convergence of the algorithm by the orthogonality $\left\langle r_{t-1}, \Phi_{\lambda_{t}}\right\rangle=\left\langle r_{t-1}-a_{t}, \Phi_{\lambda_{t}}\right\rangle=$ 0 between its latest error $\Phi_{\lambda_{t}}$ and the currently selected column vector $\Phi_{\lambda_{t}}$. However, this point only guarantees the orthogonality of the redundant vector $r_{t}$ and $\Phi_{\lambda_{t}}$, but cannot guarantee the orthogonality of r, and the selected column vector $\operatorname{set}\left(\Phi_{\lambda_{1}}, \Phi_{\lambda_{2}}, \ldots, \Phi_{\lambda_{t}}\right\}$, which makes the approximate solution obtained by MP. K terms are all sub-optimal in the sense of approximation, which is why the MP algorithm requires more iterations to ensure convergence. The OMP algorithm ${ }^{(22)}$ recursively orthogonalizes the set of selected column vectors to find the orthogonal projection $P_{t}$, and use the residual update method for $r_{t}=y-P_{t} y$ to overcome the sub-optimality of the MP algorithm.

Algorithm 2.2: OMP algorithm

- Initialize redundant vector $r_{0}=y$, index set $\Lambda_{0}=\phi$, iterative count number $t=1$;

- Find index $\lambda_{t}$ such that: 


$$
\lambda_{t}=\underset{j=1,2, \ldots, N}{\arg \min }\left|\left\langle r_{t-1}, \Phi_{j}\right\rangle\right|
$$

- Ordinance $\Lambda_{t}=\Lambda_{t-1} \cup\left\{\lambda_{t}\right\}$

- Calculate the orthogonal projection $P_{t}$ of $\left\{\Phi_{\lambda}: \lambda \in \Lambda_{t}\right\}$ into the space

- Calculate the new approximation a and redundant $r: a_{t}=P_{t} y, r_{t}=y-a_{t}$;

- $t=t+1$, if $\mathrm{t}<\mathrm{m}$ returns to step 2 ;

- To obtain estimates of $\widehat{s}_{\lambda}$ in the index $\Lambda_{m}$ membered non-zero position, and in this position, measurement vector is approximated as $a_{m}=\sum_{\lambda \in \Lambda_{m}} \widehat{s_{\lambda}} \Phi_{\lambda}$

The common short coming of OMP and MP is the non-optimality of the column vector selection mechanism relative to the new redundancy error, once the candidate column vector enters the supported candidate, it will be permanently added and will not be deleted again. It lacks the idea of "backtracking". Here "backtracking" means that in the steps of the current iteration, the column vector selected in the last iteration are still synchronized, and if they still meet the current optimal significance, they will be retained otherwise they will be eliminated as the next candidate. The significance lies in the global optimality of reconstruction can be guaranteed to the greatest extent, because the column vector that meets the greedy condition in a certain iteration cannot guarantee that they can still be reached in the subsequent iteration steps. All column vectors should be "deleted" and "added" freely. This idea has been widely used in a variety of subsequent greedy algorithms.

\section{Proposed Approach (i e Modified OMP algorithm)}

The procedure of iterative algorithm OMP can illustrate using the below steps:

Step 1: Initialized the residual $r=y$ and index set $S \widehat{g}=\varnothing$ and $\mathrm{t}=0$;

Step 2: Increased the iteration of the algorithm.

Step 3: Threshold:While the norm of residual is bigger than $\in$ and $t$ is less than $\bar{L}$, we iterated the residuals and continued the other steps. " $\in$ " will be a boundary to get a small error when finding an acceptable measurement matrix. After that, OMP would recover the original signal with high probability. Moreover, we limited the iteration number with feedback overload.

$$
p_{t}=Q^{H} r_{t}
$$

Step 5: Max probability of active path indexes determine using sparse vector $\hat{g}$. Its minimum noise, maximum correlation, and mean could provide

$$
n_{t}^{*}=\operatorname{argmax}_{j=1,2, \ldots . G}\left(\left|p_{t, j}\right|\right)
$$

Step 6: In an index set, the active paths' indices were added and augmented the index set.

$$
S_{\widehat{g}}=S_{\widehat{g}} U n_{t}^{*}
$$

Step 7: Until the iteration end, the estimation could continue after initializing the computed sparse vector.

$$
\begin{gathered}
\widehat{g} s_{\widehat{g}}=0 \\
\widehat{g} s_{\widehat{g}}=Q_{:, S_{\widehat{g}}}^{H} y
\end{gathered}
$$

Step 8: The received signal new approximation and the new residual was estimated.

$$
r=y-Q \widehat{g}
$$

Step 9: If the limit exceeds the loop breaking and the process ends, the algorithm will return to step 3 during the process while $t<\bar{L}$.

The residual $r$ is orthogonal to the $Q$ columns always. The feedback technique applies with quantizing non-zero elements of $\hat{\mathrm{g}}$ with index set and associated to the sparse interaction matrix $\hat{G}$ after the estimation sparse vector. The method of max Lloyd scalar quantizing applies as compressed sensing for quantization of $\hat{g}$. Then, $\hat{G}$ obtains with the reshaping of sparse $\hat{g}$ vector. The receiving bits are relevant to the non-zero indices of $\hat{g}$.

$$
H \approx \widehat{A}_{R} G \widehat{A}_{T}^{H}
$$




\subsection{Lloyd Scalar Quantization for Limitation}

The regions related to the codeword are categorized by the input field, which associates with each quantizer. To determine the portion rule and codebook, the quantities design to minimize the measure of overall average distortion.

To design the quantizer, two necessary conditions are required. Primarily, the center condition is needed to optimize the codebook. That means the measure of average decay over the region or the optimal codeword should select for the local mean distortion minimization. The second condition is the nearest neighbor rule for optimizing the channel space partition. All input vectors allow near to the code word that assigns to more regions or neighbors compared to another code word. These two conditions have been re-examined by the generalized Lloyd algorithm to determine the channel space partition and optimal codebook. The steps of this algorithm are mentioned below:

Step 1: The valid codebook initializes ( $\hat{\mathrm{g}})$.

Step 2: The nearest neighbor rule applies for finding the optimal regions for $\hat{\mathrm{g}}$.

Step 3: The optimal codewords determine by applying the center condition for optimal regions.

Step 4: Until convergence, these steps have continued.

The overall average distortion has reduced monotonically due to the nearest neighbor rule and center condition. The sparse channel non-zero elements can estimate the iteration. Based on the known thresholds, the sparse vector can dequantize at BS after quantization.

The number of feedback bits is the non-zero elements of the $\hat{\mathrm{g}}$ :

$$
\bar{L}=\log _{2} G+2 Q
$$

Where $2 \mathrm{Q}$ is the quantization bit number where one $\mathrm{Q}$ is real part and another is imaginary part of the CSI, and $\mathrm{G}$ is the dictionary member's multiplication (GRGT) and $\bar{L}$ is related to directly OMP algorithm for limitation of feedback bits.

\section{Results and Discussion}

The proposed modified OMP model is evaluated in this section. The evaluation parameters used in the experimental analysis are Normalized Mean Square Error and channel capacity, described in sections 4.1 and 4.2 respectively.

\subsection{Normalized Mean Square Error(NMSE) Calculation}

In this work, the recovery performance of the CS-based OMP-SQ technique, average Normalized Mean Squared Error (NMSE) and sum capacity are investigated under different quantization bits.

In literature, there are several calculation techniques for NMSE. One of them calculates NMSE between the perfect channel and the reconstructed channel. And, the other one calculates NMSE regarding the reconstructed channel. ||.|| represents the $\mathrm{L}_{2}$-norm. NMSE between the estimated channel and perfect channel can be represented as

$$
\begin{gathered}
\text { NMSE }=\frac{\|(\widetilde{H}-H)\|_{2}}{\left(\widetilde{H}_{m} \times H_{m}\right)} \\
\|(\widetilde{H}-H)\|_{2}=\frac{1}{N} \sum_{i=1}^{N}(\widetilde{H}-H)_{2}
\end{gathered}
$$

Where $\widetilde{H}$ reconstructed channel and $\mathrm{H}$ is the perfect channel, $\widetilde{H}_{m}$ represented the average reconstructed channel and $H_{m}$ represented the average of a perfect channel.

\subsection{Capacity Calculation of Reconstructed and Perfect Channel for Performance Analysis}

Shannon Capacity of a MIMO Channel:

$$
C_{r}=\log \left[\operatorname{det}\left(I_{M_{R}}+\frac{S N R}{M_{T}} \widetilde{H} \times \widetilde{H}^{H}\right)\right]
$$




$$
\begin{gathered}
C_{p}=\log \left[\operatorname{det}\left(I_{M_{R}}+\frac{S N R}{M_{T}} H \times H^{H}\right)\right] \\
C_{e r}=\log \left[\operatorname{det}\left(I_{M_{R}}+\frac{S N R}{M_{T}} H \times H_{e r}^{H}\right)\right]
\end{gathered}
$$

Where $\mathrm{C}_{r}$ is the estimated channel capacity and $\mathrm{C}_{p}$ is perfect channel capacity and $\mathrm{C}_{e r}$ is error channel capacity, and can be represented by $\mathrm{M}_{r} \times \mathrm{M}_{r}$ in identity matrix.

The capacity of a channel highly relies on the matrix structure $\mathrm{H}$. The perfect, estimated, and error channel capacities were calculated using the Eq. (14), (15), and (16) respectively and they were related to the SNR directly.

Due to the non-similarity of matrix dimensions, the normalized mean-squared error (NMSE) is used to examine the difference between non-perfect, perfect, and estimated channels.

\subsection{Simulation Results}

We consider the error-free uplink feedback channel and simulation parameters which are represented in Table 1 .

Table 1. Simulation Parameters

\begin{tabular}{lll}
\hline S.No & Parameters & metrics \\
\hline 1 & Number of Transmitting Antennas & 128 \\
2 & Number of Receiving Antennas & 2 \\
3 & Carrier frequency & $2 \mathrm{GHz}$ \\
4 & Speed of light & $3 \times 10^{8}$ \\
5 & Carrier wavelength $(\mathrm{m})$ & 0.15 \\
6 & max directional gain & $8 \mathrm{db}$ \\
7 & degree 3dB beamwidth & 55 \\
8 & front to back ratio & 30 \\
9 & Transmitting angular resolution & 140 \\
10 & Receiving angular resolution & 16 \\
\hline
\end{tabular}

Based on $\mathrm{M}_{t}$, the channel was established and is directly relevant to NMSE. The relation between $\mathrm{M}_{t}$ and NMSE can be viewed from Figure 1. While increasing the $\mathrm{M}_{t}$, NMSE is decreasing as it is expected according to Eq. (12) and (13). Also, after 256 transmitter antennas number, NMSE is maintaining the same value, so 128 is an optimum number of antennas.

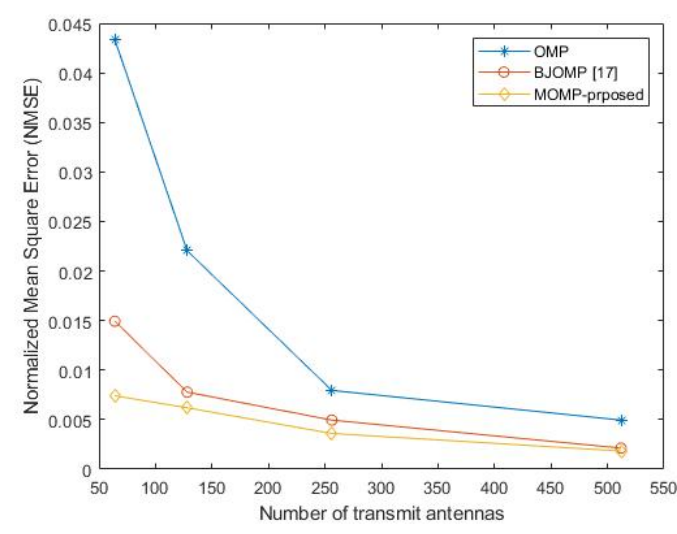

Fig 1. NMSE vs $M_{t}$ (Number of transmit antennas)

In Figure 1, the proposed OMP algorithm is compared with traditional OMP and BJOMP ${ }^{(23)}$ algorithms. The proposed method reduces the channel estimation period by sacrificing the length of observation; as a result the burden in feedback bits 
estimation has been reduced. From Figure 1, 20\% of the mean error rate is reduced when compared with the existing method reported in ${ }^{(23)}$.

The effect of the quantization level of the Lloyd algorithm has been shown in Figure 2 . The feedback channel limits the feedback burden, and decreases the NMSE using the proposed algorithm. Figure 2 represents the Signal to Noise Ratio (SNR) versus capacity.

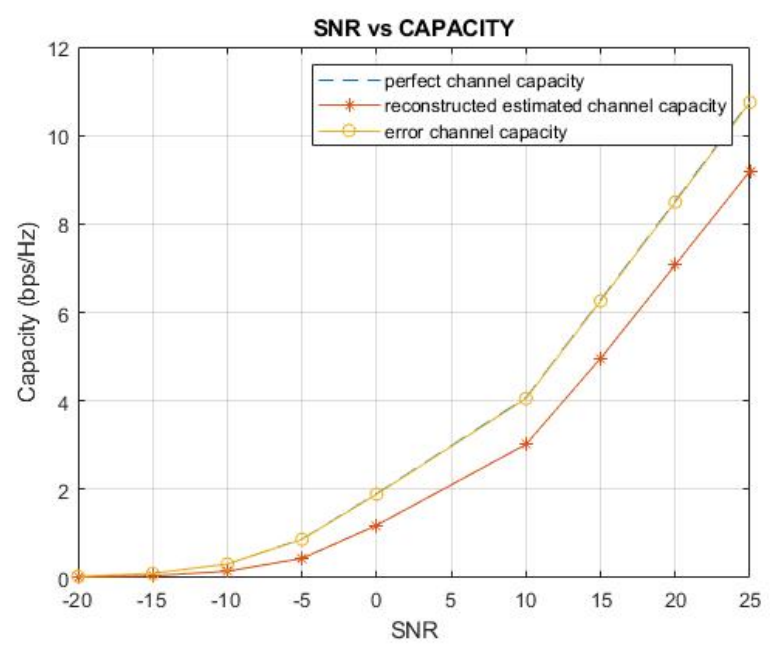

Fig 2. SNR vs Capacity

Figure 2 shows that with the increased SNR values, the capacity increases according to Eq. (14), (15),= and (16). The red line shows the estimated capacity of a channel and it is lower than the channel error. As the error reduces the channel capacity improves, the upper and lower bounds of the channel capacity are 3 and 13 bits/ $\mathrm{Hz}$ for 0 - and $25-\mathrm{dB}$ SNR. This results in a high data rate MIMO system that can be accommodated in the available bandwidth.

\section{Conclusions}

The presented approach optimizes the feedback and reduces the complexity. Our approach minimizes the delay and is well suited for massive MIMO real-time applications. This method provides the base to the research community to improve the massive MIMO system. Simulation results validate our results. The results show that feedback overhead is reduced and the normalized mean square error is reduced by $20 \%$.

\section{References}

1) Lu L, Li GY, Swindlehurst AL, Ashikhmin A, Zhang R. An Overview of Massive MIMO: Benefits and Challenges. IEEE Journal of Selected Topics in Signal Processing. 2014;8(5):742-758. Available from: https://dx.doi.org/10.1109/jstsp.2014.2317671.

2) Xu J, Zhu P, Li J, You X. Energy Efficiency Optimization for MIMO Distributed Antenna Systems With Pilot Contamination. IEEE Access. 2018;6:2415724170. Available from: https://dx.doi.org/10.1109/access.2018.2831210.

3) Choi J, Love DJ, Bidigare P. Downlink Training Techniques for FDD Massive MIMO Systems: Open-Loop and Closed-Loop Training With Memory. IEEE Journal of Selected Topics in Signal Processing. 2014;8(5):802-814. Available from: https://dx.doi.org/10.1109/jstsp.2014.2313020.

4) Han Y, Lee J, Love DJ. Compressed Sensing-Aided Downlink Channel Training for FDD Massive MIMO Systems. IEEE Transactions on Communications. 2017;65(7):2852-2862. Available from: https://dx.doi.org/10.1109/tcomm.2017.2691700.

5) Ke M, Gao Z, Wu Y, Gao X, Schober R. Compressive Sensing-Based Adaptive Active User Detection and Channel Estimation: Massive Access Meets Massive MIMO. IEEE Transactions on Signal Processing. 2020;68:764-779. Available from: https://dx.doi.org/10.1109/tsp.2020.2967175.

6) Myzhang X, Chen. Segmental channel feedback for massive MIMO with compressive sensing. Computer Technology and Development. 2017;27(6):183186.

7) Dai L, Gao Z, Wang Z. Joint channel estimation and feedback with low overhead for FDD massive MIMO systems. 2015 IEEE/CIC International Conference on Communications in China (ICCC). 2015;p. 1-6. Available from: https://dx.doi.org/10.1109/ICCChina.2015.7448660.

8) Huang W, Huang Y, Xu W, Yang L. Beam-Blocked Channel Estimation for FDD Massive MIMO With Compressed Feedback. IEEE Access. 2017;5:1179111804. Available from: https://dx.doi.org/10.1109/access.2017.2715984.

9) Shen W, Dai L, Shi Y, Shim B, Wang Z. Joint Channel Training and Feedback for FDD Massive MIMO Systems. IEEE Transactions on Vehicular Technology. 2016;65(10):8762-8767. Available from: https://dx.doi.org/10.1109/tvt.2015.2508033.

10) Schwarz S, Rupp M, Wesemann S. Grassmannian Product Codebooks for Limited Feedback Massive MIMO With Two-Tier Precoding. IEEE Journal of Selected Topics in Signal Processing. 2019;13(5):1119-1135. Available from: https://dx.doi.org/10.1109/jstsp.2019.2930890. 
11) Zheng F, Chen Y, Pang B, Liu C, Wang S, Fan D, et al. An Efficient CSI Feedback Scheme for Dual-Polarized Massive MIMO. IEEE Access. 2018;6:2342023430. Available from: https://dx.doi.org/10.1109/access.2018.2811838.

12) Sun H, Zhao Z, Fu X, Hong M. Limited Feedback Double Directional Massive MIMO Channel Estimation: From Low-Rank Modeling to Deep Learning. 2018 IEEE 19th International Workshop on Signal Processing Advances in Wireless Communications (SPAWC). 2018;p. 1-5. Available from: https://dx.doi.org/10.1109/SPAWC.2018.8446005.

13) Liao Y, Yang X, Yao H, Chen L, Wan S. Spatial correlation based channel compression feedback algorithm for massive MIMO systems. Digital Signal Processing. 2019;94:38-44. Available from: https://dx.doi.org/10.1016/j.dsp.2019.07.004.

14) Liao Y, Chen L, Hua Y, Zhang S, Shen X, Yi H. M-SAMP: A Low-complexity Modified SAMP Algorithm for Massive MIMO CSI Feedback. 2018 IEEE/CIC International Conference on Communications in China (ICCC). 2018;p. 547-552. Available from: https://dx.doi.org/10.1109/ICCChina.2018.8641150.

15) Darshankumar C, Dalwadi HB, Soni. A Novel Channel Estimation Technique of MIMO-OFDM System based on Modified Kalman Filter. Indian Journal of Science and Technology. 2016;(9):1-6. Available from: https://dx.doi.org/10.17485/ijst/2016/v9i36/97757.

16) Singh N, Shukla MK. Channel Estimation of DWT Based MIMO-OFDM System in Wireless Communication. Indian Journal of Science and Technology. 2019;12(46):01-08. Available from: https://dx.doi.org/10.17485/ijst/2019/v12i46/148006.

17) Hindy A, Mittal U, Brown T. CSI Feedback Overhead Reduction for 5G Massive MIMO Systems. 2020 10th Annual Computing and Communication Workshop and Conference (CCWC). 2020;p. 116-0120. Available from: https://dx.doi.org/10.1109/CCWC47524.2020.9031236.

18) Lee WS, Song HK. Efficient Channel Feedback Scheme for Multi-User MIMO Hybrid Beamforming Systems. Sensors. 2021;21(16):5298-5298. Available from: https://dx.doi.org/10.3390/s21165298.

19) Borges D, Montezuma P, Dinis R, Beko M. Massive MIMO Techniques for $5 G$ and Beyond-Opportunities and Challenges. Electronics. 2021;10(14):16671667. Available from: https://dx.doi.org/10.3390/electronics10141667.

20) Mashhadi MB, Gündüz D. Deep Learning for Massive MIMO Channel State Acquisition and Feedback. Journal of the Indian Institute of Science. 2020;100(2):369-382. Available from: https://dx.doi.org/10.1007/s41745-020-00169-2.

21) Yao S, Guan Q, Wang S, Xie X. Fast sparsity adaptive matching pursuit algorithm for large-scale image reconstruction. EURASIP Journal on Wireless Communications and Networking. 2018;2018(1). Available from: https://dx.doi.org/10.1186/s13638-018-1085-6.

22) Schnass K. Average Performance of Orthogonal Matching Pursuit (OMP) for Sparse Approximation. IEEE Signal Processing Letters. 2018;25(12):18651869. Available from: https://dx.doi.org/10.1109/lsp.2018.2878061.

23) Nassersadeghi M, Azghani. Channel Estimation using Block Sparse Joint Orthogonal Matching Pursuit in Massive MIMO Systems. 2021 26th International Computer Conference, Computer Society of Iran (CSICC);p. 1-5. Available from: https://dx.doi.org/10.1109/CSICC52343.2021.9420624. 